\section{Rheumatic disorders associated with immune checkpoint inhibitors: what about myositis? An analysis of the WHO's adverse drug reactions database}

As shown by Kostine $e t a l,{ }^{1}$ and recently underlined by Ceccarelli et $a l^{2}$, rheumatic inflammatory disorders induced by anticancer therapy are becoming a major concern for rheumatologists at the era of immune checkpoint inhibitors (ICIs). Beyond inflammatory arthritis, which may concern $10 \%-20 \%$ of patients, myositis represents a rare $(<1 \%)$ but potentially life-threatening event. We thus aimed at investigating the risk of ICI-related myositis in reallife setting using VigiBase, the WHO's pharmacovigilance database.

First, we analysed the myositis case associated with ICIs (AntiProgrammed Death (PD)-1, anti-Programmed Death Ligand (PDL)-1, and anti-Cytotoxic-T-Lymphocyte-Associated Protein (CTLA)-4 agents) reported to VigiBase. From 14786263 adverse drug reactions (ADRs) recorded between 1 January 2008 and 12 February 2019, we identified 54085 ICI-related ADRs including 345 myositis (0.6\%) (table 1). Among myositis cases, 85.2\% occurred with anti-PD-1 or anti-CTLA-4 monotherapies, while $14.8 \%$ with combination therapy. Lung (34.8\%) and skin cancers (34.2\%) were the most frequent indications for ICI therapy. ICIrelated myositis was more frequent in male patients, and over 65 years. The median time to onset was $4-5$ weeks ranging from 1 day to 20 months, consistently with other reports. ${ }^{3}$ Almost all ICIrelated myositis (95.3\%) were considered serious (ie, requiring at least a hospitalisation), with a fatality rate of $22.3 \%$. Myocarditis and myasthenia were associated with ICI-related myositis in $11.3 \%$ and $11.9 \%$ of cases and resulted in death in $51.3 \%$ and $26.8 \%$, respectively.

Second, using case/non-case analyses, ${ }^{4}$ we found that myositis was reported more than 17 times with ICI agents than with any other drugs (reporting OR 17.3; 95\% CI: 15.5 to 19.2). Moreover, myositis was more reported in the group of anti-PD-1/ PD-L1 monotherapy compared with anti-CTLA-4 monotherapy $(\mathrm{OR}=2.4,95 \% \mathrm{CI}: 1.6$ to 3.5$)$. Myositis was also more frequently reported in patients using ICI combination therapy versus those using ICI monotherapy (OR=1.8, 95\% CI: 1.3 to 2.4). Similar results were obtained after adjusting for potential confounders such as sex, age and reporter type.

Herein, we report a considerable outbreak of ICI-related myositis in the past years. VigiBase represents the largest medical postmarketing surveillance database allowing the study of ADRs in real conditions of use and facilitates the study of rare ADRs such as myositis, which is hardly observed in clinical trials or observational studies with limited sample size. A previous work by Anquetil et al identified 180 ICI-related myositis. ${ }^{5}$ With 165 additional cases, we report the largest series of ICI-related myositis and further characterise this unique ICI-related ADR using disproportionality (case/non-case) analyses, a method designed for early detection of pharmacovigilance signals. ${ }^{4}$ Although disproportionality estimates cannot be interpreted as risk estimates, they have been shown to be significantly correlated to risk estimates. ${ }^{6}$

Interestingly, ICI-related myositis seems to differ greatly in comparison to primitive inflammatory muscle disorders (IMDs), suggesting a novel and unique emerging autoimmune entity with specific concerns. Indeed, clinical manifestations in ICI-related myositis include frequent bulbar symptoms, ptosis and oculomotor impairment, whereas those are rare in primitive myositis, but may occur in myasthenia gravis, a disorder affecting neuromuscular junction. Hence, the association between immune-related myositis
Table 1 Characteristics of immune checkpoint inhibitor (ICI)exposed myositis cases

\begin{tabular}{|c|c|}
\hline Characteristics & ICl-exposed myositis cases $(n=345)$ \\
\hline Age (years), median (P25-P75) ( $n=265)$ & $71(63-76)$ \\
\hline \multicolumn{2}{|l|}{ Sex, $n(\%)$} \\
\hline Male & $228(69.7)$ \\
\hline Female & $99(30.3)$ \\
\hline Unknown & 18 \\
\hline \multicolumn{2}{|l|}{ Reporter type, n (\%) } \\
\hline Health professional & $293(86.2)$ \\
\hline Other & $47(13.8)$ \\
\hline Unknown & 5 \\
\hline \multicolumn{2}{|l|}{ Reporting year, $\mathrm{n}(\%)$} \\
\hline 2019 & $4(1.2)$ \\
\hline 2018 & $184(53.3)$ \\
\hline 2017 & $90(26.1)$ \\
\hline 2016 & $47(13.6)$ \\
\hline $2008-2015$ & $20(5.8)$ \\
\hline \multicolumn{2}{|l|}{ Cancer type, n (\%) } \\
\hline Lung cancer & $111(34.8)$ \\
\hline Skin cancer & $109(34.2)$ \\
\hline Melanoma & $102(32.0)$ \\
\hline Other cancers & $32(31 \%)$ \\
\hline \multicolumn{2}{|l|}{ Exposure to ICls } \\
\hline Monotherapy, n (\%) & $294(85.2)$ \\
\hline Anti-PD-1 & $252(73.0)$ \\
\hline Nivolumab & $154(44.6)$ \\
\hline Pembrolizumab & $97(28.1)$ \\
\hline Nivolumab or pembrolizumab & $1(0.3)$ \\
\hline Anti-PD-L1 & $15(4.3)$ \\
\hline Atezolizumab & $7(2.0)$ \\
\hline Avelumab & $3(0.9)$ \\
\hline Durvalumab & $5(1.4)$ \\
\hline Anti-CTLA-4 & $27(7.8)$ \\
\hline Ipilimumab & $27(7.8)$ \\
\hline Tremelimumab & $0(0.0)$ \\
\hline Combination therapy & $51(14.8)$ \\
\hline Nivolumab/ipilimumab & $49(14.2)$ \\
\hline Pembrolizumab/ipilimumab & $1(0.3)$ \\
\hline Durvalumab/tremelimumab & $1(0.3)$ \\
\hline \multicolumn{2}{|l|}{ Time to onset (days) ( $n=97$ ) } \\
\hline Median (P25-P75) & $33(19-57)$ \\
\hline Min-Max & $1-606$ \\
\hline \multicolumn{2}{|l|}{ Reported myositis term } \\
\hline Myositis & $276(80.0)$ \\
\hline Dermatomyositis & $25(7.2)$ \\
\hline Polymyositis & $20(5.8)$ \\
\hline Immune-mediated necrotising myopathy & $13(3.8)$ \\
\hline Orbital myositis & $8(2.3)$ \\
\hline Inclusion body myositis & $2(0.6)$ \\
\hline Antisynthetase syndrome & $1(0.3)$ \\
\hline \multicolumn{2}{|l|}{ Specific co-reported irAEs } \\
\hline Myocarditis & $39(11.3 \%)$ \\
\hline Myasthenia & $41(11.9 \%)$ \\
\hline Death, n (\%) & $77(22.3)$ \\
\hline
\end{tabular}

and myasthenia gravis may not be fortuitous as shown here and by others, even if specific antibodies against acetylcholine receptor may be lacking. ${ }^{7-9}$ Interestingly, the great majority of myasthenia gravis cases was not observed with anti-CTLA-4 agents, but rather with anti-PD-1/PD-L1 agents, suggesting an increased risk with the latter, in line with our proper observations. ${ }^{9}$

Of note, the majority of the reports were merely qualified as 'myositis', even if some also referred to specific entities. This may originate from reporting difficulties but questions the complex 
nosology in IMD. Actually, the classification of IMD improved considerably in the last years and now distinguishes definite entities according to clinical presentation, specific auto-antibody profiles, histological patterns, associated with distinct prognoses. If these data may be lacking in VigiBase, case reports have characterised ICI-myositis as driven by T-CD8-lymphocyte and macrophages infiltrates together with fibre necrosis, a description close to necrotising myositis. ${ }^{9}$ Reports have described circulating antibodies against antisynthetase, polymyositis-scleroderma PM-SCL), signal recognition particle (SRP) and transcription intermediary factor 1 gamma (TIF-1-gamma). ${ }^{9}$ Noteworthily, the latter is strongly associated with cancer and considered as a paraneoplastic syndrome (PNS) in this context. By analogy with other pre-existing autoimmune conditions, the use of ICIs in the context of PNS may be associated with an increased risk for immune toxicity, including PNS flare. ${ }^{10}$ Beyond these considerations, the association with other autoimmune entities may also be of interest, since myositis can also stand as a manifestation of other connective tissue disorders, such as lupus, scleroderma or Sjögren syndrome. ${ }^{11}$

Importantly, another major concern with ICI-related myositis is the strong association with myocarditis. In accordance with our results, other series revealed high prevalence of myocarditis (ranging from $15 \%$ to $32 \%$ ) in patients with ICI-related myositis, together with high lethality (up to 50\%). In another study on VigiBase, which focused on ICI-related myocarditis, musculoskeletal disorders were the most frequent concurrent complications occurring along with myocarditis. ${ }^{12}$ Thus, the increased prevalence of myocarditis may explain the higher mortality rate in ICI-related myositis compared with primitive IMD, and prompts for a systematic cardiac screening in these patients.

In conclusion, despite the limitations inherent to pharmacovigilance studies which are concerned with under-reporting, we confirm a strong signal suggesting an increasing risk of myositis associated with ICIs, especially anti-PD-1/PD-L1 agents or when these drugs were used in combination with anti-CTLA-4 agents. Prospective studies will be necessary to better investigate this risk, and better define the place of ICI-related myositis within the spectrum of IMD. In the meantime, clinicians' awareness and vigilance are needed to improve early detection and management of this unique and severe complication. In this context, the specific risk of myocarditis, a life-threatening complication, prompts to a systematic screening.

\section{Thủy Nguyễn, ${ }^{1}$ Alexandre Thibault Jacques Maria ${ }^{2},{ }^{2,3}$ Chayma Ladhari, ${ }^{1}$ Pascale Palassin, ${ }^{1}$ Xavier Quantin, ${ }^{4}$ Candice Lesage, ${ }^{5}$ Guillaume Taïeb, ${ }^{6}$ Xavier Ayrignac, ${ }^{6}$ Patricia Rullier, ${ }^{2}$ Dominique Hillaire-Buys, ${ }^{1}$ Olivier Lambotte, ${ }^{7}$ Philippe Guilpain, ${ }^{2,3}$ Jean-Luc Faillie ${ }^{1}$}

'Department of Medical Pharmacology and Toxicology, University of Montpellier, Faculty of Medicine, Montpellier, Languedoc-Roussillon, France

${ }^{2}$ Department of Internal Medicine: Multi-Organic Diseases, Local Referral Center for Systemic Autoimmune Diseases, Montpellier University Hospital, University of Montpellier, Medical School, Montpellier cedex 5, France

${ }^{3}$ IRMB Institute for Regenerative Medicine and Biotherapy, INSERM U1183, University of Montpellier, Montpellier, France

${ }^{4}$ Department of Medical Oncology, Montpellier Cancer Institute, University of Montpellier, Faculty of Medicine, Montpellier, Languedoc-Roussillon, France ${ }^{5}$ Department of Dermatology, University of Montpellier, Faculty of Medicine, Montpellier, Languedoc-Roussillon, France

${ }^{6}$ Department of Neurology, University of Montpellier, Faculty of Medicine, Montpellier, Languedoc-Roussillon, France

${ }^{7}$ Department of Internal Medicine, Hopital Bicetre, Le Kremlin-Bicetre, France

Correspondence to Dr Alexandre Thibault Jacques Maria, Internal Medicine: MultiOrganic Diseases, Local Referral Center for systemic autoimmune diseases, Montpellier University Hospital, Univ Montpellier, Medical School, Montpellier cedex 5, France; alexandremaria@hotmail.fr

Correction notice This article has been corrected since it published Online First.
The author contribution statement has been amended.

Contributors All authors meet the criteria for authorship, for their substantial contributions to the conception or design of the work, the acquisition and the interpretation of data. All authors have revised the manuscript critically and gave their final approval of the version published. They give agreement for all aspects of the work in ensuring that questions related to the accuracy or integrity of any part of the work.

Funding The authors have not declared a specific grant for this research from any funding agency in the public, commercial or not-for-profit sectors.

Disclaimer The Uppsala Monitoring Centre has provided the data, but the study results and conclusions are those of the authors and not necessarily those of the Uppsala Monitoring Centre, National Centres or WHO.

Competing interests ATJM has received fees from Abbvie, Actelion, CSL Behring, Experf, Novartis and Shire, and declares speaking fees from Astra-Zeneca and BMS in the last 5 years. PG is a medical expert for LFB (Laboratoire Français du Biofractionnement) and has received fees from Abbvie, Actelion, Boehringer Ingelheim France, Bouchara-Recordati, Novartis, Pfizer and Roche in the last 5 years. OL has received expert testimony and consultancy fees from BMS France, MSD, Astra Zeneca; consultancy fees from Genzyme, Incyte, and expert testimony for Janssen. Other authors declare that they have no conflicts of interest.

Patient consent for publication Not required.

Provenance and peer review Not commissioned; internally peer reviewed.

(c) Author(s) (or their employer(s)) 2020. No commercial re-use. See rights and permissions. Published by BMJ.

TN and ATJM contributed equally.

PG and J-LF contributed equally.

$$
\text { D) Check for updates }
$$

To cite Nguyễn T, Maria ATJ, Ladhari C, et al. Ann Rheum Dis Epub ahead of print: [please include Day Month Year]. doi:10.1136/annrheumdis-2020-217018

Received 19 January 2020

Accepted 22 January 2020

Ann Rheum Dis 2020;0:1-2. doi:10.1136/annrheumdis-2020-217018

\section{ORCID iD}

Alexandre Thibault Jacques Maria http://orcid.org/0000-0002-0868-5804

\section{REFERENCES}

1 Kostine M, Rouxel L, Barnetche T, et al. Rheumatic disorders associated with immune checkpoint inhibitors in patients with cancer-clinical aspects and relationship with tumour response: a single-centre prospective cohort study. Ann Rheum Dis 2018;77:393-8.

2 Ceccarelli F, Botticelli A, Gelibter AJ, et al. 'Immune checkpoint inhibitorinduced inflammatory arthritis persists after immunotherapy cessation' by Braaten et al: another point of view. Ann Rheum Dis 2020. doi:10.1136/ annrheumdis-2019-216867

3 Shah M, Tayar JH, Abdel-Wahab N, et al. Myositis as an adverse event of immune checkpoint blockade for cancer therapy. Semin Arthritis Rheum 2019:48:736-40.

4 Faillie J-L. Case-non-case studies: principle, methods, bias and interpretation. Therapie 2019:74:225-32.

5 Anquetil C, Salem J-E, Lebrun-Vignes B, et al. Immune checkpoint inhibitor-associated myositis. Circulation 2018;138:743-5.

6 Maciá-Martínez M-A, de Abajo FJ, Roberts G, et al. An empirical approach to explore the relationship between measures of disproportionate reporting and relative risks from analytical studies. Drug Saf 2016;39:29-43.

7 Suzuki S, Ishikawa N, Konoeda F, et al. Nivolumab-related myasthenia gravis with myositis and myocarditis in Japan. Neurology 2017;89:1127-34.

8 Mammen AL, Rajan A, Pak K, et al. Pre-Existing antiacetylcholine receptor autoantibodies and $B$ cell lymphopaenia are associated with the development of myositis in patients with thymoma treated with avelumab, an immune checkpoin inhibitor targeting programmed death-ligand 1. Ann Rheum Dis 2019;78:150-2.

9 Moreira A, Loquai C, Pföhler C, et al. Myositis and neuromuscular side-effects induced by immune checkpoint inhibitors. Eur J Cancer 2019;106:12-23.

10 Manson G, Maria ATJ, Poizeau F, et al. Worsening and newly diagnosed paraneoplastic syndromes following anti-PD-1 or anti-PD-L1 immunotherapies, a descriptive study. J Immunother Cancer 2019;7:337.

11 Ramos-Casals M, Maria A, Suárez-Almazor ME, et al. Sicca/Sjögren's syndrome triggered by PD-1/PD-L1 checkpoint inhibitors. data from the International ImmunoCancer registry (ICIR). Clin Exp Rheumatol 2019;37 Suppl 118:114-22.

12 Salem J-E, Manouchehri A, Moey M, et al. Cardiovascular toxicities associated with immune checkpoint inhibitors: an observational, retrospective, pharmacovigilance study. Lancet Oncol 2018;19:1579-89. 